\title{
Acute kidney injury during daptomycin versus vancomycin treatment in cardiovascular critically ill patients: a propensity score matched analysis
}

Philippe Gaudard ${ }^{1 *}$ (D, Marine Saour ${ }^{2}$, David Morquin ${ }^{3}$, Hélène David ${ }^{1}$, Jacob Eliet ${ }^{2}$, Maxime Villiet ${ }^{4}$, Jean-Pierre Daures ${ }^{5}$ and Pascal Colson ${ }^{2}$

\begin{abstract}
Background: Gram-positive organisms are a leading cause of infection in cardiovascular surgery. Furthermore, these patients have a high risk of developing postoperative renal failure in intensive care unit (ICU). Some antibiotic drugs are known to impair renal function. The aim of the study was to evaluate whether patients treated for Gram-positive cardiovascular infection with daptomycin (DAP) experienced a lower incidence of acute kidney injury (AKI) when compared to patients treated with vancomycin (VAN), with comparable efficacy.
\end{abstract}

Methods: ICU patients who received either DAP or VAN, prior to or after cardiovascular surgery or mechanical circulatory support, from January 2010 to December 2012, were included in this observational retrospective cohort study. We excluded patients with end stage renal disease and antibiotic prophylaxis. The primary endpoint was the incidence of AKI within the first week of treatment. Secondary endpoints were the incidence of AKI within the first 14 days of treatment, the severity of AKI including renal replacement therapy (RRT), the rates of clinical failure (unsuccessful infection treatment) and of premature discontinuation and mortality. To minimize selection bias, we used a propensity score to compare the 2 groups. Univariate and multivariate analysis were performed to determine factors associated with AKI.

Results: Seventy two patients, treated for infective endocarditis, cardiovascular foreign body infection, or surgical site infection were included (DAP, $n=28$ and VAN, $n=44)$ ). AKl at day 7 was observed in $28(64 \%)$ versus $6(21 \%)$ of the VAN and DAP patients, respectively $(p=0.001)$. In the multivariate analysis adjusted to the propensity score, vancomycin treatment was the only factor associated with AKI (Odds Ratio 4.42; 95\% Cl: 1.39-15.34; $p=0.014$ ). RRT was required for 2 (7\%) DAP patients and 13 (30\%) VAN patients, $p=0.035$. Premature discontinuation and clinical failure occurred more frequently in VAN group than in DAP group ( $25 \%$ versus $4 \%, p=0.022$ and $42 \%$ versus $12 \%$, respectively, $p=0.027$ ).

Conclusions: Daptomycin appears to be safer than vancomycin in terms of AKI risk in ICU patients treated for cardiovascular procedure-related infection. Daptomycin could be considered as a first line treatment to prevent AKI in high-risk patients.

Keywords: Vancomycin, Daptomycin, Acute kidney injury, Nephrotoxicity, Infective endocarditis, Foreign body associated infection, Cardiovascular surgery

\footnotetext{
* Correspondence: p-gaudard@chu-montpellier.fr

${ }^{1}$ PhyMedExp, University of Montpellier, CNRS, INSERM, Department of

cardiothoracic Anaesthesiology and Critical Care Medicine, CHU Montpellier,

Montpellier, France

Full list of author information is available at the end of the article
}

(c) The Author(s). 2019 Open Access This article is distributed under the terms of the Creative Commons Attribution 4.0 International License (http://creativecommons.org/licenses/by/4.0/), which permits unrestricted use, distribution, and reproduction in any medium, provided you give appropriate credit to the original author(s) and the source, provide a link to the Creative Commons license, and indicate if changes were made. The Creative Commons Public Domain Dedication waiver (http://creativecommons.org/publicdomain/zero/1.0/) applies to the data made available in this article, unless otherwise stated. 


\section{Background}

Gram-positive organisms are a leading cause of cardiac or vascular infection, particularly in patients undergoing cardiovascular surgery with foreign body implantation. These infections may be related to the surgery indication (i.e. infectious endocarditis) or to postoperative infectious complication (i.e. surgical site infection, cardiovascular device or prosthesis infection). Patients with sepsis or septic shock require an appropriate and adequate antibiotic regimen, including early administration, broad spectrum and high doses $[1,2]$. The antibiotic treatment is started often probabilistically, then adapted to isolated strains.

Acute kidney injury (AKI) is a frequent complication that occurs in 15 to $25 \%$ of patients after vascular surgery [3-6], and up to $40 \%$ of patients after cardiac surgery [7-9]. In this surgical population, AKI that requires renal replacement therapy (RRT) ranges from 1 to $6 \%[8,9]$. AKI compromises seriously short and long-term prognosis of critically ill patients, namely patients admitted in intensive care unit (ICU) with at least one organ failure, especially during sepsis [10, 11]. Several AKI risk factors have been identified including a chronic pathology of the patient such as kidney failure or diabetes, acute kidney injury related to hemodynamic disorders during surgery, including cardiopulmonary bypass, or sepsis, and the use of nephrotoxic agents such as some antibiotics, colloids or iodine contrast agents $[8,9,12,13]$. Avoiding nephrotoxic agents is therefore strongly recommended in ICU patients, to reduce the incidence of AKI, or to reduce its severity [14].

In this respect, Daptomycin, which showed good safety and efficacy in previous studies $[15,16]$, may be a safer alternative to vancomycin. In a first large validation study, non-inferiority of daptomycin for the treatment of bacteraemia and endocarditis caused by Staphylococcus aureus against standard treatment (low dose gentamycin plus vancomycin or penicillinase-resistant penicillin) was demonstrated with a lower rate of renal dysfunction [17]. However, the criteria used to define renal dysfunction was not the currently accepted definition for AKI issued by the Kidney Disease: Improving Global Outcomes (KDIGO) consortium [14]. Moreover, there is some controversy regarding vancomycin nephrotoxicity when used in continuous intravenous infusion $[12,18]$. To our knowledge, no study has compared the nephrotoxicity of daptomycin and vancomycin in ICU patients yet.

The aim of this cohort study was to assess whether the use of daptomycin, was associated to a lower incidence of AKI than vancomycin in cardiovascular ICU patients, with similar efficacy.

\section{Methods}

\section{Study design}

This is a retrospective observational study with a propensity score adjustment to reduce the bias of selection for a comparative analysis between two antibacterial treatments used in routine care. The ethics committees ("CPP Sud Méditerranée IV", NQ-2015-05-03 and the "CCTIRS", $\left.\mathrm{N}^{\circ} 15.670\right)$ approved the study. The requirement for informed patient consent was waived since the nature of the study was retrospective. The data collection was authorized by the "CNIL" (NDR-2015 - 643).

\section{Targeted population}

From January 2010 to December 2012, patients who were admitted to a cardiothoracic and vascular surgical ICU and who received either daptomycin (DAP group) or vancomycin (VAN group), identified from the delivery drug list of the hospital pharmacy, were screened for analysis.

The following criteria were required for inclusion: (i) Patient older than 18 years; (ii) Suspected or proven cardiac, vascular or profound surgical site infection with Grampositive cocci (GPC) methicillin-resistant (MR) strains (including probabilistic treatment for patients with acquisition of MR risk factors); (iii) Treatment duration greater than or equal to $48 \mathrm{~h}$ (at least 2 doses of daptomycin administered or 2 days of vancomycin infusion); (iv) Antibiotic treatment started in peri-operative (from $48 \mathrm{~h}$ before the onset of surgery) or in postoperative period (during ICU stay). Patients with prophylaxis indication of antibiotics, kidney disease on chronic dialysis or acute onset of RRT before initiation of DAP or VAN treatment, or staphylococcus pneumonia were excluded.

\section{Interventions}

The indication for a probabilistic or documented treatment with broad spectrum antibiotics against GPC followed institutional protocols and national recommendations for the specific population of patients admitted to ICU with sepsis or septic shock in the context of cardiovascular surgery or cardiogenic shock. Infectious endocarditis means infection on native or prosthetic valve or pacemaker leads. Surgical site infection is an infection located on the cutdown tissue or profound structures, i.e. sternal infection or mediastinitis. Ventricular assist device (VAD) provides a long-term mechanical circulatory support and only profound device-related infections occurring during the postoperative period of the implantation were considered in this study (excluding driveline or delayed infections). Vascular graft infection requires usually a probabilistic treatment after surgical samples during graft replacement, which is a complex and haemorrhagic surgery. Catheter related infections were identified by bacteraemia and positive culture of the removed catheter with the same strain and were aggressively treated in a population with cardiac or vascular prosthetic materials. The other conditions to treat were an anterior mediastinitis or a septic shock in waiting of documentation. Cardiovascular foreign body infection was defined by an infection related to intra-cardiac devices or prothesis or 
vascular graft with artificial tissue with development of bacterial biofilm.

Daptomycin was administered at a dose of $8 \mathrm{mg} / \mathrm{kg}$ in thirty-minutes intravenous infusion every $24 \mathrm{~h}$ in patients without severe impairment of kidney function or every $48 \mathrm{~h}$ in case of GFR below $30 \mathrm{ml} / \mathrm{min} / \mathrm{m}^{2}$. The creatine-kinase (CK) level was measured before the initiation of DAP and at least once a week to assess the occurrence of muscular toxicity defined by an increase of CK up to 3-fold the upper superior limit without any evidence of member ischaemia.

Vancomycin intravenous treatment was initiated by a loading dose of $30 \mathrm{mg} / \mathrm{kg}$ in $1 \mathrm{~h}$ and followed by a continuous maintenance infusion dosing between 15 and 30 $\mathrm{mg} / \mathrm{kg} / \mathrm{d}$. The VAN dose was adapted to achieve a target serum vancomycin steady-state concentration of 20-30 $\mathrm{mg} / \mathrm{L}$ assessed by a daily pharmacologic monitoring (therapeutic drug monitoring).

\section{Data collection}

Demographic data, and main clinical characteristics were collected. Renal function was assessed by daily measurement of creatinine and urinary output monitoring in ICU. The need for RRT was left to the decision of the physician in charge of the patient, mainly because of threatening metabolic disorders (metabolic acidosis, hyperkalaemia) or fluid overload. The AKI stage was established during the first 7 days and 14 days after the initiation of the studied treatment.

The factors associated to renal function impairment before the antibiotic treatment were collected for the calculation of the propensity score: severity score as the Simplified Acute Physiology Score II (SAPS II) at ICU admission and the Sequential Organ Failure Assessment (SOFA) score at initiation of treatment, history of chronic renal failure (creatinine clearance below $50 \mathrm{ml} /$ min), creatinine and AKI before treatment initiation (defined as baseline), circulatory shock related to sepsis or heart failure (defined as systolic arterial pressure below $90 \mathrm{mmHg}$ and refractory to fluid challenge with evidence of end-organ hypoperfusion like oliguria, alteration of mental status or high plasma lactate), need of cardiopulmonary bypass for surgery (within $48 \mathrm{~h}$ around the antibiotic initiation), use of other nephrotoxic agents (iodine contrast, aminoglycoside, ciclosporin), documentation of GPC bacteraemia.

\section{Study endpoints}

The primary endpoint was the incidence of AKI within the first week of treatment as defined by KDIGO consensus [14].

The secondary endpoints were chosen to better characterize AKI and describe the outcome. The incidence of AKI within the first 14 days of treatment and the maximal decrease of GFR (estimated by CKD-EPI formula) during treatment were also reported. Besides KDIGO classification, severe renal failure was estimated as stage 2 or 3 of the AKI or a reduction in glomerular filtration rate (GFR) of more than $50 \%$, and from incidence and duration of RRT. Clinical treatment failure, defined by either persistent positive cultures, worsening of clinical status, death due to initial infection, or relapse after the end of treatment was assessed in case of documented GPC infection. Incidence of premature discontinuation of treatment was defined when treatment was stopped because of adverse event or clinical failure except death. Mortality rate was calculated at day 28 and month 6 .

\section{Statistical analysis}

In a previous study [17], the incidence of renal dysfunction was estimated at 12\% with daptomycin and 35\% with standard therapy. However, a higher incidence of renal failure was expected in ICU population, as much as twice the incidence observed in the reference study $[10,19]$. Therefore, we assumed that AKI may occur in $60 \%$ VAN patients, meaning that a reduction to $20 \%$ in DAP group would be significant for a risk at $5 \%$ and a power equal to $85 \%$, provided 52 patients (at least 26 in both groups) were included.

Qualitative variables were expressed in percentages and compared with the Chi-square test or Fisher's exact test. Quantitative normally distributed variables were expressed as means and standard deviations and assessed with the Student's t-test. Medians and 25th to 75th percentiles defined quantitative not normally distributed data, for which differences were analysed by the Wilcoxon-Mann-Whitney test. $P$ values below the 0.05 level were considered to indicate statistical significance.

Since treatments were not randomized, we used the propensity score method for primary endpoint analysis. For this, we included the covariates potentially related to treatment and outcome (chronic kidney disease, cardiopulmonary bypass, GPC bacteraemia, heart failure, and shock) in a multivariate logistic model explaining the choice of treatment. The propensity score was built from the selected significant covariates (chronic kidney disease, cardiopulmonary bypass, GPC bacteraemia) and was used in the second model as an adjustment covariate. After matching on the propensity score, we checked that the covariates were statistically balanced between the two treatment groups. Then, we modelled the effect of the treatment on the main endpoint (AKI within first week of treatment) by selecting the covariates associated to this result, with a threshold of significance to $15 \%$ in a univariate analysis, and by including in the multivariate logistic model the propensity score as an additional adjustment variable.

The calculations were done under $\mathrm{R}$ software [20] by the team EA2415 of the University of Montpellier. 


\section{Results}

During the 36-month period of inclusion, among the 143 patients on the pharmacy delivery list for DAP or VAN treatment, 72 patients (28 DAP and 44 VAN) were selected for the study following application of inclusion and exclusion criteria (Fig. 1). After matching to the propensity score, 16 patients of each group were paired. Baseline demographic and clinical characteristics before and after matching are summarized in Table 1. After propensity score matching, characteristics were balanced between the two groups except a significantly higher proportion of patients with heart failure in the DAP group (75\% versus $25 \%, p=0.012)$.

The reasons for ICU admission (detailed on Table 2) were different between DAP and VAN groups $(p=$ 0.004 ) with more vascular surgery for VAN patients and more cardiologic indications for DAP patients but the rate of cardiac surgery was similar. The primary infectious diseases (reported on Table 2) were distributed slightly differently between groups with more vascular graft infection in the VAN group and more VAD-related infection in the DAP group $(p=0.045)$. Cardiovascular foreign body infection concerned $51 \%$ of patients without difference between DAP $(n=16 ; 57 \%)$ and VAN ( $n=21 ; 48 \%), p=0.436$. A GPC infection was documented in 52 patients (72\%), 26 in each group, including different strains of staphylococci isolated in $55 \%$ of infections (Table 2).

Daptomycin was administered every $24 \mathrm{~h}$ in 24 patients $(86 \%)$ or every $48 \mathrm{~h}$ in 4 patients $(14 \%)$, with an initial dose of 7.9 [6.6-9.6] $\mathrm{mg} / \mathrm{kg}$. Daptomycin doses during treatment course ranged from 7.8 [6.3-9.4] to 8.4 [7.1-9.7] $\mathrm{mg} / \mathrm{kg}$. Four DAP patients experienced an asymptomatic increase in creatine kinase, all resolved without consequence or interruption of treatment but by decreasing the dose of daptomycin or increasing the interval of administration. The vancomycin median loading dose in $1 \mathrm{~h}$ was 26.7 [18.5-29.7] $\mathrm{mg} / \mathrm{kg}$ and the continuous intravenous infusion ranged from 21.7 [13.9-26.7] to 29.4 [26.8-33.1] $\mathrm{mg} / \mathrm{kg}$ daily. In the VAN group, at least one vancomycin

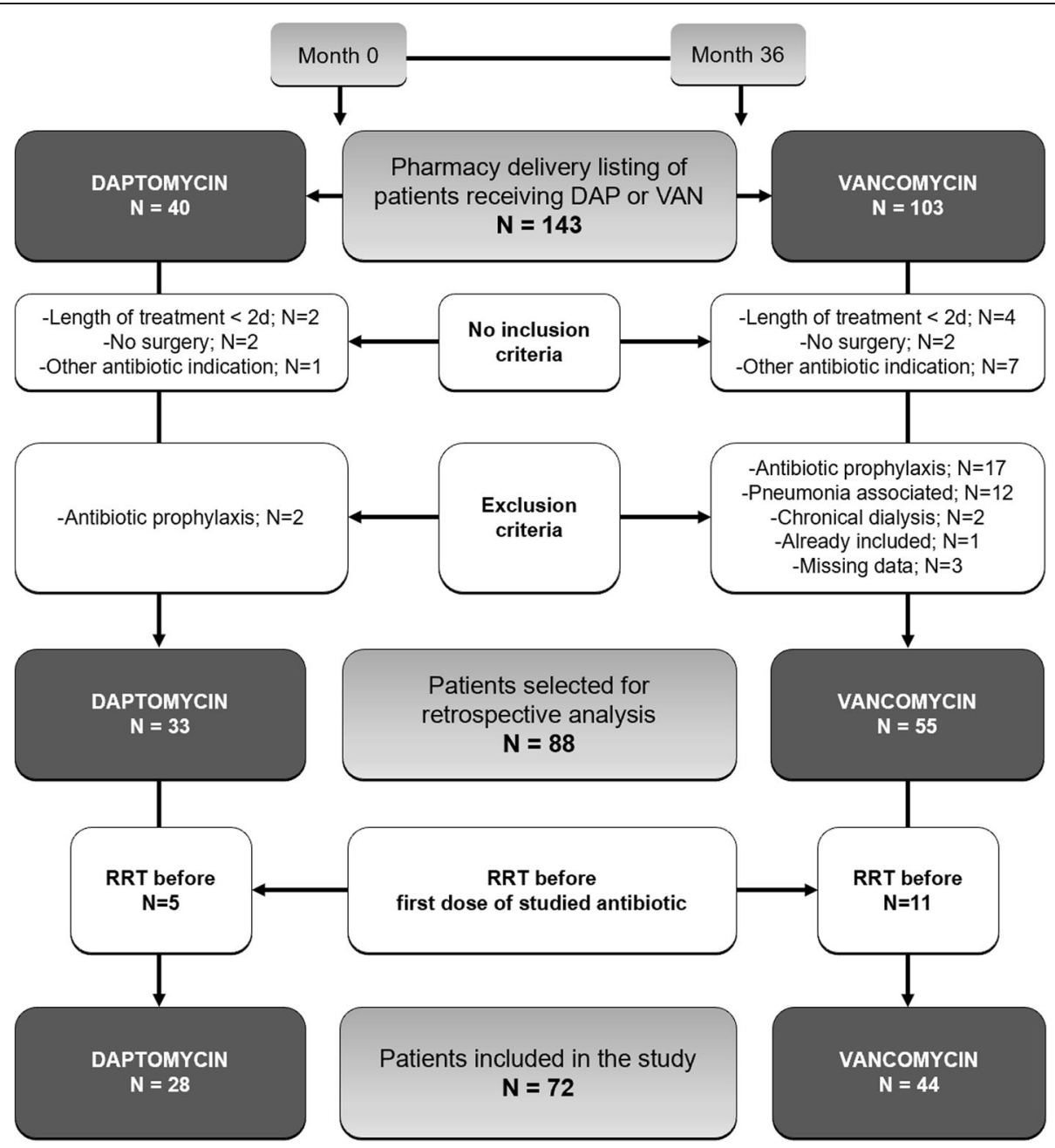

Fig. 1 Flow chart of the patient selection process. DAP, daptomycin; VAN, vancomycin; RRT, renal replacement therapy 
Table 1 Baseline demographic and clinical characteristics of patients before and after propensity score matched analysis

\begin{tabular}{|c|c|c|c|c|c|c|}
\hline \multirow[b]{2}{*}{ Characteristics } & \multicolumn{3}{|l|}{ Before matching } & \multicolumn{3}{|c|}{ Propensity score matched } \\
\hline & $\operatorname{DAP}(N=28)$ & $\operatorname{VAN}(N=44)$ & $p$ & $\operatorname{DAP}(N=16)$ & $\operatorname{VAN}(N=16)$ & $p$ \\
\hline Male sex, n (\%) & $17(61)$ & $34(77)$ & .215 & $10(63)$ & $11(69)$ & 1 \\
\hline Age, y & $60.0[52.3-70.5]$ & $63.0[54.0-75.3]$ & .206 & $56.0[49.5-69.3]$ & $65.0[49.0-78.0]$ & .086 \\
\hline Weight, kg & $74.0[61.5-80.5]$ & $72.0[64.5-80.0]$ & .827 & $71.5[61.5-79.3]$ & $75.0[63.0-85.3]$ & .383 \\
\hline Height, cm & 170 [163-173] & 167 [165-175] & .821 & $170[165-174]$ & 167 [161-169] & .179 \\
\hline History of $\mathrm{CRcl}<50 \mathrm{ml} / \mathrm{min}, \mathrm{n}(\%)$ & $8(28.6)$ & $5(11.4)$ & .125 & $2(12.5)$ & $2(12.5)$ & 1 \\
\hline History of diabetes mellitus, n (\%) & $10(35.7)$ & $11(25.0)$ & .478 & $4(25.0)$ & $6(37.5)$ & .704 \\
\hline History of hypertension & $10(35.7)$ & $23(52.3)$ & .258 & $5(31.3)$ & $8(50.0)$ & .473 \\
\hline Heart failure (EF < 50\%), n (\%) & $18(64.3)$ & $16(36.4)$ & .038 & $12(75.0)$ & $4(25.0)$ & .012 \\
\hline CPB during surgery, $\mathrm{n}(\%)$ & $9(32.1)$ & $25(56.8)$ & .071 & $7(43.8)$ & $7(43.8)$ & 1 \\
\hline VAD, n (\%) & $7(25)$ & $7(15.9)$ & .519 & $5(31.3)$ & $1(6.3)$ & .172 \\
\hline Circulatory shock at baseline, n (\%) & $8(28.6)$ & $21(47.8)$ & .171 & $4(25.0)$ & $9(56.3)$ & .149 \\
\hline Others nephrotoxic agents ${ }^{\mathrm{a}}, \mathrm{n}(\%)$ & $18(64.3)$ & $29(65.9)$ & .910 & $9(56.3)$ & $11(68.7)$ & .716 \\
\hline SAPS II score at ICU admission & $44.5[35.3-57.8]$ & $45.5[34.0-57.8]$ & .894 & $51.0[38.5-61.5]$ & $48.0[35.0-57.0]$ & .984 \\
\hline SOFA score at baseline & $5[2-9]$ & $6[3-9]$ & .388 & $4[2-7]$ & $6[3-8]$ & .281 \\
\hline Serum creatinine at baseline, $\mu \mathrm{mol} / \mathrm{l}$ & $110[77-142]$ & 99 [72-134] & .699 & $87[64-126]$ & 94 [69-124] & .440 \\
\hline AKI at baseline, $\mathrm{n}(\%)$ & $7(25.0)$ & $13(29.5)$ & .881 & $2(12.5)$ & $6(37.5)$ & .220 \\
\hline Previous antibiotic treatment, n (\%) & $19(67.8)$ & $24(54.5)$ & .381 & $8(50)$ & $8(50)$ & 1 \\
\hline GPC bacteraemia, n (\%) & $16(57.1)$ & $12(27.3)$ & .022 & $11(68.8)$ & $10(62.5)$ & .710 \\
\hline
\end{tabular}

Quantitative data are expressed as median [interquartile range]. Baseline is the time of treatment initiation

${ }^{a}$ Within 2 days before or after initiation of treatment

$D A P$ daptomycin, VAN vancomycin, $C R C l$ creatinine clearance, $E F$ ejection fraction, $C P B$ cardiopulmonary bypass, VAD ventricular assist device, SAPS Simplified

Acute Physiology Score, SOFA Sequential Organ Failure Assessment, AKI acute kidney injury, GPC Gram positive cocci

serum concentration was below $20 \mathrm{mg} / \mathrm{L}$ in $43 \%$ of patients or above $30 \mathrm{mg} / \mathrm{L}$ in $52 \%$. The treatment duration was longer for DAP than for VAN, with a median of 16 [6-31] versus 8 [4-14] days $(p=0.021)$, respectively.

The incidence of AKI at day 7 (primary endpoint) was significantly higher with vancomycin $(64 \%, n=28)$ than with daptomycin $(21.4 \%, n=6), p=0.001$ respectively. Similar results were observed at day 14 (Fig. 2).

Factors associated with 7-day AKI in univariate analysis are reported in Table 3. The multivariate logistic regression model, adjusted to the propensity score (which includes chronic kidney disease, cardiopulmonary bypass and GPC bacteraemia covariates), showed that only vancomycin treatment remained significantly associated with the occurrence of AKI with an Odds Ratio of 4.42 (95\%CI: 1.39 to 15.34), $p=0.014$ (Table 3).

AKI was more severe in VAN group than in DAP patients as assessed by incidence of AKI stage 2 or 3 or GFR decrease of more than $50 \%$ and RRT requirement and duration (Table 3).

Clinical failure of initial antibiotic therapy occurred more frequently with vancomycin than with daptomycin, namely in 11 out of 26 VAN patients (42\%) and 4 out of 26 DAP patients (15\%), with GPC documented infection, $p=0.032$ ). Premature treatment discontinuation was not significantly different between DAP and VAN groups (2 (7\%) versus 11
$(25 \%)$, respectively, $p=0.055)$. Several changes were related to vancomycin (11 cases / 44 vs $2 / 28, p=0.055)$ including 6 cases of switch from vancomycin to daptomycin. Two failure cases of daptomycin treatment resulted in a switch to vancomycin (occurrence of secondary staphylococci pneumonia, acquisition of resistance).

The ICU and hospital lengths of stay were similar in both groups (Table 4). Mortality was high in this cohort, with no significant difference at month 6 between the VAN and DAP groups (Table 4). The survival curves were not significantly different on a Kaplan Meier analysis (Fig. 3).

\section{Discussion}

Our study compared the impact of antibiotic choice against Gram-positive strains on AKI incidence in ICU patients after cardiovascular surgery, mechanical circulatory support or invasive cardiac procedure. AKI was more frequent and AKI severity higher in patients treated with vancomycin than in patients treated with daptomycin. AKI defined according to KDIGO consensus was 4-fold more likely during the first week of treatment with vancomycin than with daptomycin.

AKI incidence in ICU is about $40 \%$, ranging from 20 to $55 \%$ in various large cohort studies $[10,19]$, with an ICU mortality rate of approximately $40 \%$. Likewise, AKI 
Table 2 Description of medical context and infectious disease

\begin{tabular}{|c|c|c|c|}
\hline Characteristics & $\mathrm{DAP}(N=28)$ & $\operatorname{VAN}(N=44)$ & $p$ \\
\hline Reason for ICU admission & & & .004 \\
\hline Cardiac surgery, n (\%) & $15(53.6)$ & $26(59.1)$ & - \\
\hline Vascular surgery, $\mathrm{n}(\%)$ & $2(7.1)$ & $14(31.8)$ & - \\
\hline Interventional cardiology or short-term mechanical circulatory support, n (\%) & $9(32.1)$ & $4(9.4)$ & - \\
\hline Mediastinitis, n (\%) & $2(7.1)$ & $0(0)$ & - \\
\hline \multicolumn{3}{|l|}{ Primary infectious disease } & .045 \\
\hline Infective endocarditis, n (\%) & $8(28.6)$ & $15(34.1)$ & - \\
\hline Vascular graft infection, n (\%) & $2(7.1)$ & $12(27.3)$ & - \\
\hline VAD infection, n (\%) & $4(14.3)$ & $0(0)$ & - \\
\hline Surgical site infection, n (\%) & $5(17.9)$ & $6(13.6)$ & - \\
\hline Catheter-related infection, n (\%) & $5(17.9)$ & $4(9.1)$ & - \\
\hline Miscellaneous, n (\%) & $4(14.3)$ & $7(15.9)$ & - \\
\hline \multicolumn{3}{|l|}{ Microbiological identification } & .002 \\
\hline MSSA, n (\%) & $7(25.0)$ & $4(9.1)$ & - \\
\hline MRSA, n (\%) & $4(14.3)$ & $3(6.8)$ & - \\
\hline CNS, n (\%) & $13(46.4)$ & $9(20.5)$ & - \\
\hline Enterococcus, n (\%) & $1(3.6)$ & $4(9.1)$ & - \\
\hline Other Gram-positive cocci, n (\%) & $1(3.6)$ & $6(13.6)$ & - \\
\hline Other strains or non-documented, $\mathrm{n}(\%)$ & $2(7.1)$ & $18(40.1)$ & - \\
\hline
\end{tabular}

$P$-values for Fisher exact test. DAP daptomycin, VAN vancomycin, ICU intensive care unit, VAD ventricular assist device, MSSA methicillin-susceptible Staphylococcus aureus, MRSA methicillin-resistant Staphylococcus aureus, CNS coagulase negative staphylococci

after cardiovascular surgery, is one of the most common and serious clinical issue, associated with high morbidity and mortality rates [21]. Multiple risk factors and mechanisms have been described such as preoperative renal dysfunction, diabetes mellitus, hypertension, heart

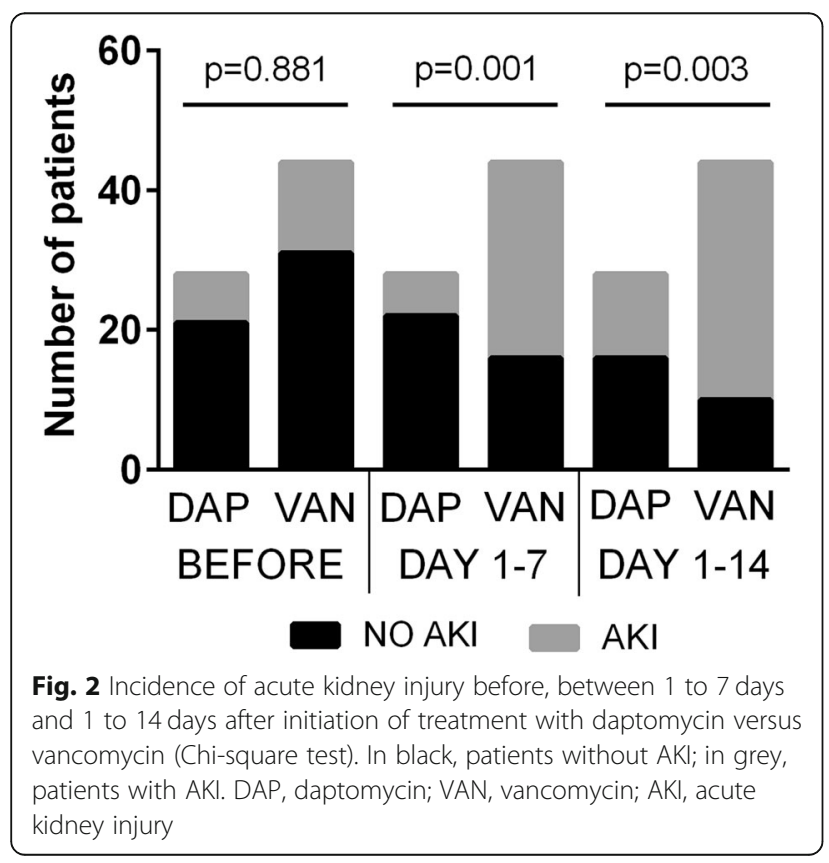

failure, anaemia or inflammatory response to $\mathrm{CPB}[3,8$, 22]. On top of that, nephrotoxic agents may contribute to AKI development, therefore alternative treatments or their discontinuation are strongly recommended whenever it is possible [14].

Our analysis suggests that daptomycin is a less nephrotoxic agent than vancomycin. These results are in agreement with previous observations [23, 24]. In a randomized trial, Fowler et al. found that a renal dysfunction at the end of treatment involved $11 \%$ of patients taking daptomycin versus $26 \%$ of patients under standard treatment, i.e. low dose gentamicin plus vancomycin or anti-staphylococcal penicillin [17]. The reduction in incidence of AKI observed in Fowler's study (57\%) is very similar to the reduction observed in the present study (67\%).

The lower incidence of AKI in daptomycin group than in the vancomycin group was observed despite the use of continuous infusion of vancomycin. Continuous infusion has been reported to be less nephrotoxic than intermittent administration [12, 25, 26]. Nevertheless, the nephrotoxicity of vancomycin remains a serious concern in high risk patients even with continuous administration [18, 27]. In a randomized controlled trial involving 100 high-risk patients, a switch from vancomycin to alternative strategies failed to prevent AKI compared to maintenance of vancomycin $(32.7 \%$ versus 31.4\%) [28]. These results suggest that even short-term 
Table 3 Covariate factors associated with AKI development in univariate and stepwise multivariate logistic regression analysis adjusted to the propensity score

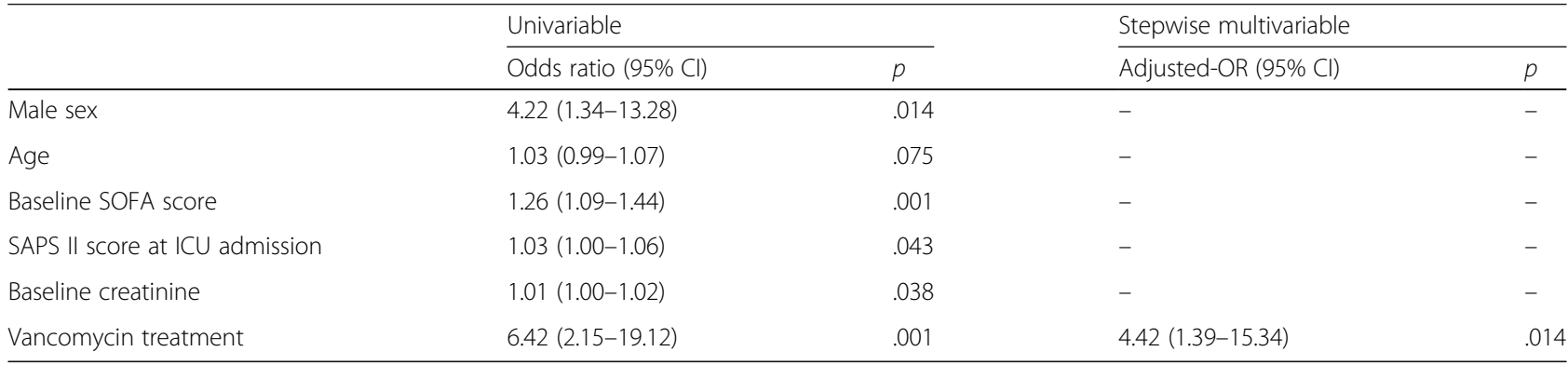

All variables related to AKI (within 7 days after drug initiation) in univariate analysis, defined by $p<0.15$ are reported, excepted those resumed in the propensity score. Variables with $p \geq 0.15$ were not included into the model. All variables entered into the backward stepwise multivariate model including the propensity score were not independently associated with AKI excepted the treatment with vancomycin. AKI acute kidney injury, SOFA Sepsis-Related Organ Failure Assessment, SAPS Simplified Acute Physiology Score

initial exposition to vancomycin may be able to impair renal function.

In the present study, observation of RRT exposure in the two groups showed lower indication and shorter duration of RRT in DAP group than in VAN group (Table 4). These results suggest further that daptomycin is less nephrotoxic than vancomycin. Only few studies have addressed the issue of daptomycin or vancomycin treatment during RRT [23].

The VAN group was exposed to numerous overdoses as assessed by serum concentration, in $52 \%$ of patients, which may have contributed to the nephrotoxic effect of vancomycin. Inversely, despite the administration of a vancomycin dose consistent with a strict institutional protocol to achieve a target steady-state serum concentration of $20-30 \mathrm{mg} / \mathrm{L}$ as recommended [25, 29], low vancomycin levels were also observed $(43 \%)$ and may have contributed to therapeutic failure. Moreover, untimely discontinuation of initial treatment represented
$18.1 \%$ of the total population including 6 cases of switch from vancomycin to daptomycin. In patients with non-pulmonary infection due to MR strains (MR Staphylococcus aureus, MR coagulase negative staphylococci, Enterococcus species), daptomycin given at $6 \mathrm{mg} / \mathrm{kg}$ every $24 \mathrm{~h}$ is considered equivalent in efficacy compared to standard treatment with vancomycin for some authors [17, 30, 31]. However, in a recent retrospective and matched cohort of patients with MRSA bloodstream infection, clinical failure was significantly higher in the vancomycin cohort than in the daptomycin cohort (45.0\% versus $29.0 \%)$ [32]. Similar results have been reported in other retrospective studies [24, 33]. These observations highlight the difficulty of achieving a narrow, effective and safe therapeutic target with vancomycin in ICU. Of note, reduced susceptibility to daptomycin may be induced in some strains of Staphylococcus aureus by prior vancomycin exposure [30].

Table 4 Severity and ICU management of AKI and outcomes

\begin{tabular}{|c|c|c|c|}
\hline Variables & $\mathrm{DAP}(N=28)$ & $\operatorname{VAN}(N=44)$ & $p$ \\
\hline \multicolumn{4}{|l|}{ AKI severity and management } \\
\hline AKI stage 2 or 3 , or GFR decrease $>50 \%$ & $7(25.0)$ & $25(56.8)$ & .008 \\
\hline Maximal variation of GFR except RRT $(N=57), \%$ & $-6[-46$ to +13$]$ & $-31[-49$ to -8$]$ & .055 \\
\hline RRT initiated during treatment, $\mathrm{n}(\%)$ & $2(7.1)$ & $13(29.5)$ & .022 \\
\hline Proportion of ICU days with RRT ${ }^{\mathrm{a}}$, \% & $0[0-0]$ & $0[0-13]$ & .032 \\
\hline \multicolumn{4}{|l|}{ Outcomes } \\
\hline Muscular toxicity (CK > 3xUSL), n (\%) & $4(14.3)$ & 0 & \\
\hline Hypersensitivity manifestation, n (\%) & 0 & $1(2)$ & \\
\hline Length of ICU stay ${ }^{\mathrm{a}, \mathrm{b}}(N=53)$, days & $9[4-28]$ & $12[5-25]$ & .827 \\
\hline Length of in-hospital stay ${ }^{a, b}(N=41)$, days & 47 [19-62] & 39 [23-52] & .543 \\
\hline 28-day mortality ( $N=71), \mathrm{n}(\%)$ & $3(10.7)$ & $11(25.6)$ & .124 \\
\hline 180-day mortality ( $N=69), \mathrm{n}(\%)$ & $10(35.7)$ & $22(53.7)$ & .142 \\
\hline
\end{tabular}

Quantitative data are expressed as median [interquartile range], except otherwise specified

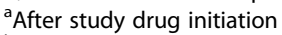

${ }^{\mathrm{b}}$ Censored for inpatient mortality

DAP daptomycin, VAN vancomycin, GFR estimate glomerular filtration rate, RRT renal replacement therapy, AKI acute kidney injury, CK creatine kinase, USL upper superior limit, ICU intensive care unit 


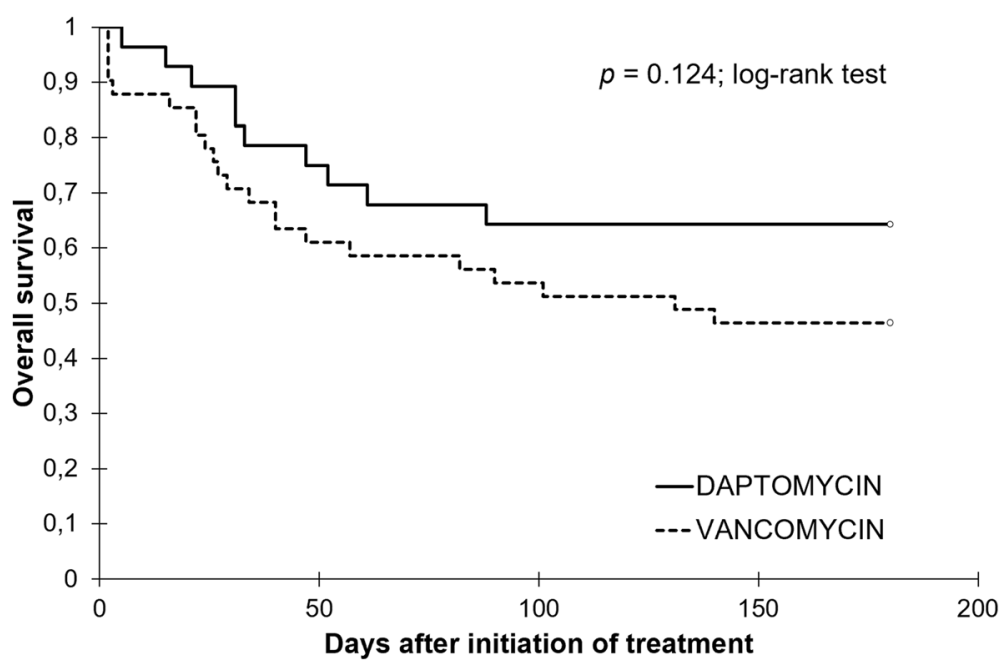

Fig. 3 Kaplan Meier analysis of day-180 survival for surgical ICU patients with cardiovascular infection treated by daptomycin (full line) or vancomycin (dotted line)

Daptomycin was used at high doses as recommended in order to limit the emergence of daptomycin resistance and to speed up bactericidal activity [34, 35]. Drug registry data suggest that doses up to $8 \mathrm{mg} / \mathrm{kg}$ every $24 \mathrm{~h}$ are well-tolerated and effective [36, 37]. A recently published review concluded that strong experimental evidence suggests that higher doses (e.g. $8-10 \mathrm{mg} / \mathrm{kg}$ ) of daptomycin should be used for MRSA and enterococcal infections in critically ill patients as well as in bacteraemia and endocarditis [38]. No serum concentration of daptomycin was available, but the treatment regimen seemed appropriate to achieve appropriate antibacterial efficacy, at least not less than vancomycin, with a rather simple protocol, without pharmacological monitoring.

\section{Limitations}

Due to the retrospective design, selection bias and missing data may have influenced the results. The minimal inhibitory concentrations of vancomycin for MRSA strains were not available for most of the patients. Furthermore, no follow up after hospital discharge was organized. The inclusion period was chosen during the first 3 years of daptomycin use in our unit, since we observed an increase in its indication instead of vancomycin in our routine practice after this period. Although the sample of this study is limited and there is always a risk of hidden bias, the absence of a previous or expected randomized trial in this area allows us to retain this result. Despite these limitations, both groups were balanced with respect to baseline characteristics (especially for severity scores SAPS II and SOFA). The striking difference in AKI incidence between both groups, added to the adjustment with the propensity score, suggest these limitations cannot overrule the clinical relevance of the results.

\section{Conclusions}

Vancomycin resulted in significantly more AKI and increased RRT exposure compared to daptomycin in a cardiovascular ICU population. In addition, daptomycin seems well appropriate to maintain an effective and sustained treatment for severe infections, such as endocarditis or cardiac devices and vascular graft infections, specifically in the context of AKI.

\section{Abbreviations}

AKl: Acute kidney injury; Cl: Confidence interval; DAP: Daptomycin; GFR: Glomerular filtration rate; GPC: Gram positive cocci; ICU: Intensive care unit; KDIGO: Kidney disease improving global outcome; MR: Methicillin resistance; OR: Odds ratio; RRT: Renal replacement therapy; SAPS II: Simplified acute physiology score II; SOFA: Sequential organ failure assessment; VAN: Vancomycin

\section{Acknowledgements}

Dr. Brigitte Calvet and Dr. Patrick Davignon contributed to the design of this study and made it possible to get started by helping to find funding from Novartis.

\section{Funding}

The study was supported by a grant from Novartis Pharma exclusively dedicated to obtain an independent research assistant for the data collection and an external statistical analysis.

\section{Availability of data and materials}

The datasets used and/or analysed during the current study are available from the corresponding author on reasonable request.

\section{Authors' contributions}

PG was involved in the conception of the study, collection, analysis and interpretation of data, drafting and critical review of the manuscript. JPD was involved in the design of the study and statistical analysis. PC was involved in the design of the study, interpretation and critical review of the manuscript. MV and DM contributed to the selection of patients and the interpretation of data. MS, HD and JE contributed to the data collection and interpretation. All authors had substantial input to the drafting and review of the manuscript and approved the final version prior to publication. 


\section{Ethics approval and consent to participate}

The study was approved by the independent ethics committee "CPP Sud Méditerranée IV" (NQ-2015-05-03) and the "CCTIRS" ( $\left.\mathrm{N}^{\circ} 15.670\right)$. The requirement for informed consent was waived. An information letter was sent to patients requesting their non-opposition consent. This procedure and this anonymous data collection were authorized by the CNIL (NDR-2015-643).

\section{Consent for publication}

Not applicable.

\section{Competing interests}

The authors declare that they have no competing interests.

\section{Publisher's Note}

Springer Nature remains neutral with regard to jurisdictional claims in published maps and institutional affiliations.

\section{Author details}

'PhyMedExp, University of Montpellier, CNRS, INSERM, Department of cardiothoracic Anaesthesiology and Critical Care Medicine, CHU Montpellier, Montpellier, France. ${ }^{2}$ Department of cardiothoracic Anaesthesiology and Critical Care Medicine, CHU Montpellier, Montpellier, France. ${ }^{3}$ Infectious and Tropical Diseases Department, CHU Montpellier, Montpellier, France. ${ }^{4}$ Clinical Pharmacy Department, CHU Montpellier, Montpellier, France. ${ }^{5}$ Laboratory of Biostatistics and Epidemiology EA2415, University Institute for Clinical Research, Montpellier, France.

Received: 18 January 2019 Accepted: 9 May 2019

Published online: 20 May 2019

\section{References}

1. Corona A, Bertolini G, Lipman J, et al. Antibiotic use and impact on outcome from bacteraemic critical illness: the BActeraemia study in intensive care (BASIC). J Antimicrob Chemother. 2010;65:1276-85.

2. Kumar A, Roberts D, Wood KE, et al. Duration of hypotension before initiation of effective antimicrobial therapy is the critical determinant of survival in human septic shock. Crit Care Med. 2006:34:1589-96.

3. Ellenberger C, Schweizer A, Diaper J, et al. Incidence, risk factors and prognosis of changes in serum creatinine early after aortic abdominal surgery. Intensive Care Med. 2006;32:1808-16

4. Tallgren M, Niemi T, Pöyhiä R, et al. Acute renal injury and dysfunction following elective abdominal aortic surgery. Eur J Vasc Endovasc Surg. 2007;33:550-5.

5. Godet $\mathrm{G}$, Fleron $\mathrm{M}$, Vicaut $\mathrm{E}$, et al. Risk factors for acute postoperative renal failure in thoracic or thoracoabdominal aortic surgery: a prospective study Anesth Analg. 1997;85:1227-32.

6. Kashani K, Steuernagle JH 4th, Akhoundi A, et al. Vascular surgery kidney injury predictive score: a historical cohort study. J Cardiothorac Vasc Anesth. 2015;29: 1588-95.

7. Saydy N, Mazine A, Stevens LM, et al. Differences and similarities in risk factors for postoperative acute kidney injury between younger and older adults undergoing cardiac surgery. J Thorac Cardiovasc Surg. 2018;155:256-65.

8. Mariscalco G, Lorusso R, Dominici C, et al. Acute kidney injury: a relevant complication after cardiac surgery. Ann Thorac Surg. 2011;92:1539-47.

9. Rosner MH, Okusa MD. Acute kidney injury associated with cardiac surgery. Clin J Am Soc Nephrol. 2006;1:19-32.

10. Nisula S, Kaukonen KM, Vaara ST, et al. Incidence, risk factors and 90-day mortality of patients with acute kidney injury in Finnish intensive care units: the FINNAKI study. Intensive Care Med. 2013;39:420-8.

11. Peters E, Antonelli M, Wittebole X, et al. A worldwide multicentre evaluation of the influence of deterioration or improvement of acute kidney injury on clinical outcome in critically ill patients with and without sepsis at ICU admission: results from the intensive care over nations audit. Crit Care. 2018;22:188.

12. Hanrahan TP, Harlow G, Hutchinson J, et al. Vancomycin-associated nephrotoxicity in the critically ill: a retrospective multivariate regression analysis. Crit Care Med. 2014; 42:2527-36.

13. Cosgrove SE, Vigliani GA, Fowler VG Jr, et al. Initial low-dose gentamicin for Staphylococcus aureus bacteremia and endocarditis is nephrotoxic. Clin Infect Dis. 2009;:48:713-21

14. Kellum JA, Lameire N, Guideline Work KDIGOAKI. Group. Diagnosis, evaluation, and management of acute kidney injury: a KDIGO summary (part 1). Crit Care. 2013;17:204.
15. Dvorchik BH, Brazier D, DeBruin MF, et al. Daptomycin pharmacokinetics and safety following administration of escalating doses once daily to healthy subjects. Antimicrob Agents Chemother. 2003;47:1318-23.

16. Arbeit RD, Maki D, Tally FP, et al. The safety and efficacy of daptomycin for the treatment of complicated skin and skin-structure infections. Clin Infect Dis. 2004;38: $1673-81$.

17. Fowler VG Jr, Boucher HW, Corey GR, et al. Daptomycin versus standard therapy for bacteremia and endocarditis caused by Staphylococcus aureus. N Engl J Med. 2006; 355:653-65.

18. Spapen HD, Janssen van Doom $K$, Diltoer M, et al. Retrospective evaluation of possible renal toxicity associated with continuous infusion of vancomycin in critically ill patients. Ann Intensive Care. 2011;1:26.

19. Bellomo R, Kellum JA, Ronco C, et al. Acute kidney injury in sepsis. Intensive Care Med. 2017;43:816-28.

20. R Development Core Team. R: A language and environment for statistical computing. Vienna, Austria: R Foundation for Statistical Computing; 2014. ISBN 3900051-07-0. http://www.R-project.org

21. Lassnigg A, Schmidlin D, Mouhieddine M, et al. Minimal changes of serum creatinine predict prognosis in patients after cardiothoracic surgery: a prospective cohort study. J Am Soc Nephrol. 2004;15:1597-605.

22. Corral-Velez V, Lopez-Delgado JC, Betancur-Zambrano NL, et al. The inflammatory response in cardiac surgen: an overview of the pathophysiology and clinical implications. Inflamm Allergy Drug Targets. 2015;13:367-70.

23. Kullar R, McClellan I, Geriak M, et al. Efficacy and safety of daptomycin in patients with renal impairment: a multicenter retrospective analysis. Pharmacotherapy. 2014; 34:582-9.

24. Moise PA, Culshaw DL, Wong-Beringer A, et al. Comparative effectiveness of vancomycin versus Daptomycin for MRSA bacteremia with vancomycin MIC >1 mg/L: a multicenter evaluation. Clin Ther. 2016;38:16-30.

25. Cataldo MA, Tacconelli E, Grilli E, et al. Continuous versus intermittent infusion of vancomycin for the treatment of gram-positive infections: systematic review and meta-analysis. J Antimicrob Chemother. 2012;67:17-24.

26. Hao JJ, Chen H, Zhou JX. Continuous versus intermittent infusion of vancomycin in adult patients: a systematic review and meta-analysis. Int J Antimicrob Agents. 2016; 47:28-35.

27. DiMondi VP, Rafferty K. Review of continuous-infusion vancomycin. Ann Pharmacother. 2013;47:219-27.

28. Carreno JJ, Kenney RM, Divine G, et al. Randomized controlled trial to determine the efficacy of early switch from vancomycin to vancomycin alternatives as a strategy to prevent nephrotoxicity in patients with multiple risk factors for adverse renal outcomes (STOP-NT). Ann Pharmacother. 2017;51:185-93.

29. Cristallini $\mathrm{S}$, Hites $\mathrm{M}$, Kabtouri H, et al. New regimen for continuous infusion of vancomycin in critically ill patients. Antimicrob Agents Chemother. 2016;60:4750-6.

30. Thwaites GE, Edgeworth G-KE, et al. Clinical management of Staphylococcus aureus bacteraemia. Lancet Infect Dis. 2011;11:208-22.

31. Leonard SN, Rybak MS. Evaluation of vancomycin and daptomycin against methicillin-resistant Staphylococcus aureus and heterogeneously vancomycinintermediate $S$. aureus in an in vitro pharmacokinetic/pharmacodynamic model with simulated endocardial vegetations. J Antimicrob Chemother. 2009;63:155-60.

32. Claeys KC, Zasowski EJ, Casapao AM, et al. Daptomycin improves outcomes regardless of vancomycin MIC in a propensity-matched analysis of methicillinresistant Staphylococcus aureus bloodstream infections. Antimicrob Agents Chemother. 2016;60:5841-8.

33. Weston A, Golan Y, Holcroft C, et al. The efficacy of daptomycin versus vancomycin for methicillin-resistant Staphylococcus aureus bloodstream infection in patients with impaired renal function. Clin Infect Dis. 2014;58:1533-9.

34. Wu G, Abraham T, Rapp J, et al. Daptomycin: evaluation of a high-dose treatment strategy. Int J Antimicrob Agents. 2011;38:192-6.

35. Rose WE, Rybak MJ, Kaatz GW, et al. Evaluation of daptomycin treatment of Staphylococcus aureus bacterial endocarditis: an in vitro and in vivo simulation using historical and current dosing strategies. J Antimicrob Chemother. 2007;60: 334-40

36. Moise PA, Hershberger E, Amodio-Groton Ml, et al. Safety and clinical outcomes when utilizing high-dose ( $>$ or $=8 \mathrm{mg} / \mathrm{kg}$ ) daptomycin therapy. Ann Pharmacother. 2009:43:1211-9.

37. Kullar R, Davis SL, Levine DP, et al. High-dose daptomycin for treatment of complicated gram-positive infections: a large, multicenter, retrospective study. Pharmacotherapy. 2011;31:527-36.

38. Heidary M, Khosravi AD, Khoshnood S, et al. Daptomycin. J Antimicrob Chemother. 2018:73:1-11. 